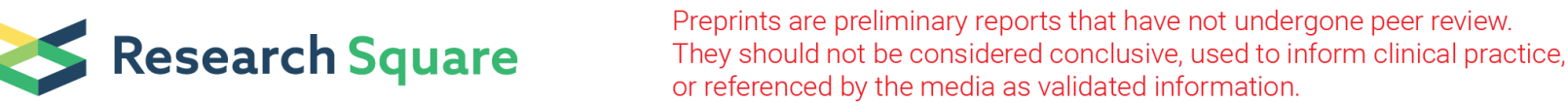

\section{Response rate and diagnostic accuracy of early PET/CT during neo-adjuvant therapies in oesophageal adenocarcinoma: protocol for a systematic review}

Jacqueline Jeffries

National Imaging Academy of Wales

Bernadette Coles

Velindre Cancer Centre

Kevin Bradley

Wales Diagnostic and Research Positron Emission Tomography Imaging Centre (PETIC)

Alex Holborow

National Imaging Academy of Wales

Elizabeth Smyth

Cambridge University Hospitals NHS Foundation Trust

Emiliano Spezi ( $\nabla$ espezi@cardiff.ac.uk)

Cardiff University https://orcid.org/0000-0002-1452-8813

Kieran Foley

Velindre Cancer Centre https://orcid.org/0000-0002-1299-1759

\section{Protocol}

Keywords: oesophageal neoplasms, positron-emission tomography, treatment response, systematic review, meta-analysis

Posted Date: August 12th, 2020

DOI: https://doi.org/10.21203/rs.3.rs-16782/v2

License: (9) This work is licensed under a Creative Commons Attribution 4.0 International License. Read Full License 


\section{Abstract}

Background Patients with potentially curable oesophageal adenocarcinoma have a staging positron emission tomography (PET) examination combined with a computed tomography (CT) to assess locoregional and distant disease. Although only $20-30 \%$ of patients are suitable for surgical resection, the majority receive neo-adjuvant therapy (chemotherapy with or without radiotherapy) before their operation. However, less than $25 \%$ experience any clinically meaningful benefit from the neo-adjuvant therapy. A repeat PET/CT after one cycle of treatment can assess for early metabolic response but remains exploratory. Patients without an early response could be offered alternative treatment strategies. The purpose of this systematic review and meta-analysis is to estimate the early response rate defined by $\mathrm{PET} / \mathrm{CT}$, its diagnostic accuracy and explore associated factors. Methods Primary studies reporting response rates and diagnostic accuracy of PET/CT will be identified from MEDLINE, Embase, Cochrane Library, Scopus, Web of Science, International Clinical Trials Registry Platform (ICTRP) Search Portal and ClinicalTrials.gov databases. Peer-reviewed randomised control trials, observational cohort, crosssectional and case-control studies reporting original response rate data, published from 2005 onwards will be included. Studies recruiting mixed cohorts and with PET/CT repeated after more than one neoadjuvant treatment cycle will be excluded. The reference standard will be pathological response, defined by either validated Becker or Mandard tumour regression grade (TRG) classifications. The primary outcome will be metabolic response rate after one neo-adjuvant treatment cycle, defined by a reduction in maximum standardised uptake value (SUVmax) of 35\%. Secondary outcome will be the sensitivity and specificity of early metabolic response to predict pathological response at this SUVmax reduction threshold. Pooled early response rate, sensitivity and specificity will be calculated using a random effects model with data extracted from selected studies. Heterogeneity between studies, risk of bias and methodological quality will be assessed. Discussion This systematic review and meta-analysis will identify and synthesise evidence to determine early response rates to neo-adjuvant therapies and the corresponding diagnostic accuracy of PET/CT to guide future clinical trials. This strategy could identify patients that will not respond to neo-adjuvant therapy and to offer this group alternative treatment

strategies. Systematic review registration: PROSPERO (registration number CRD42019147034)

\section{Background}

The incidence of oesophageal cancer is increasing worldwide, with more than 450,000 patients diagnosed each year [1]. The prognosis of oesophageal cancer is poor, especially in locally advanced and metastatic disease [2]. Despite only $20-30 \%$ of patients being suitable for surgical management [3], the majority of these receive neo-adjuvant therapy which aims to reduce the volume of disease prior to resection, and improve survival.

A number of neo-adjuvant therapy trials have shown an overall survival benefit over surgery alone [4]. The neo-adjuvant chemotherapy Medical Research Council OE02 and Adjuvant Gastric Infusional Chemotherapy (MAGIC) and peri-operative chemotherapy ACCORD-07 trials have shown significant benefit over surgery alone [2,5-7]. Similarly, the Chemoradiotherapy for Esophageal Cancer followed by 
Surgery Study (CROSS) trial showed improved survival benefit of neo-adjuvant chemoradiotherapy over surgery alone [8, 9]. More recently, perioperative chemotherapy with FLOT (5-fluorouracil (5-FU), Leucovorin, Oxaliplatin and Docetaxel) was established as the new standard-of-care for patients with operable oesophago-gastric cancer [10] in the UK, but neo-adjuvant chemoradiotherapy is still commonly used in Europe and the USA.

Positron-emission tomography combined with computed tomography (PET/CT) is now an established investigation in the routine staging pathway of oesophageal cancer [11]. 18F-fluorodeoxyglucose (FDG) is the radioactive isotope used for oesophageal cancer staging. The main advantage of PET/CT is its greater sensitivity for undetected metastases on $\mathrm{CT}$, which changes management in a significant number of patients [12], thus preventing them from undergoing major surgical intervention for little potential benefit. Research focus has now been placed on the role of PET/CT to predict pathological response earlier in the treatment pathway [13]. Pathological response to neo-adjuvant treatment is a good surrogate marker for overall survival [8], therefore prediction of which patients will have a favourable pathological response is important.

Different classifications for pathological response exist, and each are prone to subjective user error [14]. The two main histopathological classifications are the Becker [15] and Mandard [16] tumour regression grade (TRG) classifications, which are used throughout the Western world. Becker TRG 1 (either 1a, no residual tumour, or $1 \mathrm{~b},<10 \%$ residual tumour) defines a good clinical response. Similarly, Mandard TRG 1 or 2 (no residual cancer, or rare residual cancer cells, respectively) represent a good response, and was significantly associated with overall survival in a multi-centre study of oesophageal adenocarcinoma patients [17].

Metabolic response, defined by PET/CT imaging, is thought to predict underlying pathological response. In PET/CT, a quantitative metric called the maximum standardised uptake value (SUVmax) represents the highest recorded uptake of FDG within the tumour [18]. Preliminary work demonstrated that a reduction in SUVmax of 35\% from baseline PET/CT to a repeat PET/CT after one cycle of neo-adjuvant chemotherapy was predictive of pathological response, and was associated with overall survival [19]. This threshold was validated [20], and is commonly used in studies assessing early metabolic response.

Subsequent Phase II trials [21] have shown promising results using this early response threshold of $35 \%$. However, phase III trials are required to test the application of early metabolic response assessment in larger populations of oesophageal adenocarcinoma patients before it is adopted into clinical practice. To adequately power these trials, an accurate estimation of early response rate in this patient population is required. This systematic review and meta-analysis will aim to synthesise the evidence to provide these data.

Early metabolic response assessment has the potential to change clinical management by differentiating patients with an early response from those who are resistant to treatment. The latter group could potentially be offered an alternative neo-adjuvant therapy or simply omit the remaining cycles and proceed directly to surgery, thereby reducing the exposure to potential harmful side-effects, and reducing 
the chance of progression during the interval before surgery. Although the potential clinical benefits are apparent, studies often include heterogenous populations with mixed histological cell type and varying neo-adjuvant regimens. Furthermore, consensus regarding the optimal threshold to define metabolic response has not been agreed. Here, we report the protocol for a systematic review and, if feasible, a meta-analysis of early response rate to neo-adjuvant therapies in patients with oesophageal adenocarcinoma.

\section{Objectives}

The primary objective of the study is to systematically review the available literature reporting early response rate, defined by PET/CT after one cycle of neo-adjuvant therapy, in patients with oesophageal adenocarcinoma. The secondary objective is to review the literature reporting diagnostic accuracy;

specifically, the sensitivity and specificity of early PET-CT, using a threshold reduction in SUVmax of 35\%, to predict pathological response. We will aim to perform meta-analyses. Potential explanatory factors associated with early response rates will be explored using meta-regression. The protocol is registered with PROSPERO (registration number CRD42019147034) and has been reported in accordance with the PRISMA-P guidelines (additional file 1 - PRISMA-P checklist) [22].

\section{Methods}

\section{Eligibility_criteria}

Studies will be selected for review according to the eligibility criteria below.

\section{Study design and participants}

This review will include randomised control trials, observational cohort, cross-sectional and case-control studies reporting original response rate data in adult human participants. Participants will be patients with biopsy-proven oesophageal, or gastro-oesophageal junction adenocarcinoma (confirmed by histopathologist), who have been treated with neo-adjuvant chemotherapy or neo-adjuvant chemoradiotherapy prior to surgical resection and had an early PET/CT examination (after one-cycle of neoadjuvant therapy). (Fig. 1) Recurrent oesophageal adenocarcinoma will not be included. Studies of patients with histology other than adenocarcinoma and those who did not have an early PET/CT examination will be excluded. Studies with mixed patient cohorts will also be excluded.

\section{PET/CT examinations}

The radioactive isotope FDG must have been used for the PET/CT examination. Studies using other radioactive isotopes will be excluded but if the article contains FDG data, attempt will be made to extract these data. The SUVmax must have been measured by an appropriately trained and experienced professional. SUVmax is defined as the voxel with the highest SUV value within the tumour [18]. 
The threshold for response classification, defined in terms of the percentage reduction in SUVmax, must be pre-specified at $35 \%$. The reference standard will be the pathological TRG, defined by validated pathological classification systems Becker [15] or Mandard [16]. Occasionally, patients will progress during neo-adjuvant therapy and no longer be suitable for surgery. Attempt will be made to capture these data also.

$\underline{\text { Searches }}$

\section{Electronic Searches}

A comprehensive search strategy using text words and controlled vocabulary has been designed using MEDLINE (OVID). (Table 1)

Table 1. Search terms and strategy 


\begin{tabular}{|c|c|}
\hline \# & Searches \\
\hline 1 & Esophageal Neoplasms/ \\
\hline 2 & ((oesophag* or esophag*) adj (neoplas* or adenocarcinoma or tumo?r*)).tw. \\
\hline 3 & 1 or 2 \\
\hline 4 & exp Adenocarcinoma/ \\
\hline 5 & (oesophag* or esophag*).tw. \\
\hline 6 & 4 and 5 \\
\hline 7 & 3 or 6 \\
\hline 8 & Positron Emission Tomography Computed Tomography/ \\
\hline 9 & (PET-CT or PET CT).tw. \\
\hline 10 & ((early or interim or ad interim or endpoint or timepoint) adj2 (PET or FDG-PET)).tw. \\
\hline 11 & Positron-Emission Tomography/ \\
\hline 12 & (PET or FDG-PET or 18-FDG).tw. \\
\hline 13 & Fluorodeoxyglucose F18/ \\
\hline 14 & or/8-13 \\
\hline 15 & 7 and 14 \\
\hline 16 & exp Treatment Outcome/ \\
\hline 17 & exp "Predictive Value of Tests" / \\
\hline 18 & exp "sensitivity and specificity" / \\
\hline 19 & exp Disease-Free Survival/ \\
\hline 20 & exp Prognosis/ \\
\hline 21 & (sensitivity or specificity).tw. \\
\hline 22 & ((treatment or therapeutic) adj1 (response or outcome*)).tw. \\
\hline 23 & (predict* or prognos*).tw. \\
\hline 24 & ((tumo?r or metabolic) adj2 response).tw. \\
\hline 25 & tumo?r glucose.tw. \\
\hline 26 & (glucose adj (standard uptake or SUV)).tw. \\
\hline 27 & Glucose/ \\
\hline 28 & or $/ 16-27$ \\
\hline 29 & 15 and 28 \\
\hline 30 & limit 29 to (english language and yr="2005 -Current") \\
\hline 31 & randomized controlled trial.pt. \\
\hline 32 & controlled clinical trial.pt. \\
\hline 33 & randomized.ab. \\
\hline 34 & placebo.ab. \\
\hline 35 & drug therapy.fs. \\
\hline 36 & randomly.ab. \\
\hline 37 & trial.ab. \\
\hline 38 & groups.ab. \\
\hline 39 & or/31-38 \\
\hline 40 & exp animals/ not humans.sh. \\
\hline 41 & 39 not 40 \\
\hline 42 & epidemiologic studies/ \\
\hline 43 & exp case control studies/ \\
\hline 44 & exp cohort studies/ \\
\hline 45 & Case control.tw. \\
\hline 46 & exp Longitudinal Studies/ \\
\hline 47 & exp Retrospective Studies/ \\
\hline 48 & exp Prospective Studies/ \\
\hline 49 & (cohort adj (study or analys* or studies)).tw. \\
\hline 50 & exp Follow-Up Studies/ \\
\hline 51 & (Follow up adj (study or studies)).tw. \\
\hline 52 & exp Cross-Sectional Studies/ \\
\hline 53 & (observational adj (study or studies)).tw. \\
\hline 54 & (Longitudinal or Retrospective or Cross sectional).tw. \\
\hline 55 & or $/ 42-54$ \\
\hline 56 & 41 or 55 \\
\hline
\end{tabular}


This strategy will be translated and run in the following electronic databases: MEDLINE [OVID], Embase [OVID], Cochrane Library [Wiley], Cumulative Index of Nursing and Allied Health Literature (CINAHL) [Ebsco], Scopus [Elsevier], Web of Science [Thomson Reuter], International Clinical Trials Registry Platform (ICTRP) Search Portal [World Health Organisation] and ClinicalTrials.gov.

The search will be limited to articles published in English from 2005 onwards, because 3D PET became integrated into most PET/CT scanners providing more standardisation in SUVmax from this timepoint onwards [13]. Study filters for randomised control trials and observational study types will be applied. Reference lists of all eligible studies will be checked and undergo citation tracking for additional eligible studies.

\section{Selection process}

Following the systematic search described above, all titles and abstracts will be screened by two independent authors against the defined eligibility criteria. Duplicate items will be identified and one of the copies deleted prior to screening. Full text articles will be obtained for all studies that meet the criteria. In cases of disagreement following screening of titles and abstracts, a third author will be asked to review and decide upon the suitability of the study. Reasons for exclusion will be recorded. A flowchart will be used to summarise the numbers of included and excluded studies at each stage of the selection process.

\section{Data management and extraction process}

The results of the screening process will be shared between the reviewers using an output file that can be imported into Mendeley Desktop 1.19.4. Authors will be instructed to create a new library to keep the screened studies separate. The full-text articles will be included in the output file.

Relevant data will be extracted from the final set of eligible articles. Data will be inputted into a Microsoft Excel 365 spreadsheet designed specifically for this review (additional file 2 -

SystRev_Dataltems_Extraction). Two independent authors will extract the relevant data from the articles. In cases of disagreement, a third author will be asked to review the article and decide upon the data to be recorded. Articles reporting findings from duplicate sets of patients will be combined and extracted as a single study.

\section{$\underline{\text { Data items }}$}

a. Patient characteristics; number of patients included, age, gender, tumour location, neo-adjuvant regimen, pathological response rate at surgery, length of survival.

b. Study characteristics; primary author, publication year, study dates, country of study, study design, number of centres, length of time between early PET and surgery, length of time between early PET 
and surgery, conclusions of study, risk of bias.

c. PET/CT characteristics; timing of early PET/CT (days after treatment inception), type of scanner and acquisition (including PET reconstruction method), length of fasting before injection, time between FDG injection and PET, PET quantification method, interpreter(s), threshold criteria for defining response, proportion of patients with early response after one cycle of neo-adjuvant therapy.

d. Diagnostic accuracy data; true positives, false positives, false negatives, true negatives, sensitivity, specificity, positive predictive value, negative predictive value.

\section{$\underline{\text { Outcomes }}$}

The primary outcome of the systematic review will be the early response rate on PET/CT after one cycle of neo-adjuvant therapy. The secondary outcome will be diagnostic accuracy of early metabolic response, defined as the sensitivity and specificity of PET/CT to predict pathological response using the $35 \%$ SUVmax reduction threshold. The reference standard for pathological response will be the tumour regression grade (TRG). Pathological responders will be classified at pathology as having a Mandard TRG 1-2 or Becker TRG 1, and non-responders as Mandard TRG 3-5 or Becker TRG 2-3.

\section{Quality assessment and risk of bias}

The methodological quality of eligible studies will be assessed using two pre-specified quality assessment tools; the Newcastle Ottowa scale (modified for this review) [23] and the Quality Assessment of Studies of Diagnostic Accuracy Included in Systematic Reviews (QUADAS-2) criteria [24]. The Newcastle Ottowa scale will be used for the primary objective and QUADAS-2 for the secondary objective. The overall components of the quality assessment for each study will be reported using the star-rating system of the Newcastle Ottowa scale. Studies with no stars for any of the individual components of the NOS (selection, comparability and outcome assessment) will not be included in the quantitative synthesis. (additional file 3 - Modified NOS) For QUADAS-2, perceived quality will be graded low, high or unclear risk. The question "were uninterpretable and/or intermediate test results reported?" has been added to the QUADAS-2 checklist. (additional file 4 - QUADAS-2) The strength of the overall weight of evidence for both primary and secondary outcomes will be judged using the Grading of Recommendations Assessment, Development and Evaluation (GRADE) working group methodology [25].

\section{Data synthesis}

If the studies are sufficiently homogenous in their design, outcome assessment and follow-up, we will conduct a meta-analysis using a random effects model (DerSimonian and Laird [26]) using the current version of $R$ ( $R$ Foundation for statistical computing, Vienna, Austria) [27]. We will combine the percentage of patients with early metabolic response in each individual study to estimate a pooled early response rate with a $95 \%$ confidence interval $(\mathrm{Cl})$. 
The sensitivity and specificity of early PET/CT using the 35\% threshold level to define metabolic response in individual studies will be calculated and subjected to meta-analysis, if feasible. The results of the individual studies will be displayed with a receiver operator curve (ROC) curve, and a weighted symmetric summary ROC (sROC) curve with a $95 \% \mathrm{Cl}$ will be computed [28].

We will assess heterogeneity between specific study estimates using the inconsistency index $\left(\mathrm{I}^{2}\right.$ statistic [29]). If heterogeneity is considerable $\left(1^{2}>75 \%\right)$ and the $p$ value $<0.1$, quantitative data synthesis will not be performed [22]. Publication bias will be assessed by visually inspection or funnel plots [30].

We will also investigate sources of heterogeneity between studies using meta-regression subgroup analyses by stratifying original co-variates according to methodological quality (Newcastle Ottowa and QUADAS-2 score), sample size, PET injection time, neo-adjuvant therapy regimen, type of neo-adjuvant therapy and TRG classification used. The time interval between injection of FDG and scanning can affect the SUVmax of the tumour [31]. Differences in pathological response rates have been reported depending on the type of neo-adjuvant therapy used $[8,10]$. Also, the effect of the TRG classification system will be explored.

\section{Discussion}

Despite significant improvements in overall survival with neo-adjuvant therapies, less than half of patients who are treated are cured of their cancer $[2,8]$. Therefore, identification of patients who are unlikely to benefit from treatment either before or at an early timepoint during neo-adjuvant treatment is desirable.

PET/CT is commonly used in oesophageal cancer staging because the tumours frequently demonstrate metabolic activity. Metabolic response following chemotherapy on early PET/CT has been described as an imaging biomarker in this disease. Currently, the most validated metric for PET metabolic response is a reduction in SUVmax of 35\% on day 15 after one cycle of cisplatin-5FU based chemotherapy [19-21].

However, there are several unanswered questions regarding the use of early PET as a biomarker, particularly with chemotherapy regimens having evolved over the past decade. Specifically, the early response rates to different neo-adjuvant therapies are unknown and the optimum threshold for defining metabolic response remains controversial.

Possible limitations of this review include the validation of early response. This distinction is largely based on imaging findings alone at present because a lack of gold standard histopathological reference standard exists, in contrast to the time following completion of neo-adjuvant therapy, when the most direct comparison can be made with surgical resection. Other possible limitations include a possible paucity of evidence from the primary literature and significant heterogeneity between studies which would hamper the ability to perform a meta-analysis. 
This systematic review and meta-analysis will build on results from previous reviews $[13,32]$ and specifically focus on the response rate and diagnostic accuracy of early PET/CT in oesophageal adenocarcinoma. The findings of this review will inform design of future clinical trials by demonstrating the proportion of patients that have an early response after one cycle of neo-adjuvant therapy and determine the pooled diagnostic accuracy of early PET/CT to predict pathological response.

\section{Abbreviations}

PET Positron emission tomography

CT Computed tomography

$\mathrm{PET} / \mathrm{CT} \quad$ PET combined with CT

FDG Fluorodeoxyglucose

CINAHL Cumulative Index of Nursing and Allied Health Literature

ICTRP International Clinical Trials Registry Platform

MAGIC Medical Research Council OE02 and Adjuvant Gastric Infusional Chemotherapy

CROSS Chemoradiotherapy for Esophageal Cancer followed by Surgery Study

5-FU 5-fluorouracil

FLOT 5-FU, Leucovorin, Oxaliplatin and Docetaxel

${ }^{18} \mathrm{~F} \quad$ 18-Fluorine

SUVmax Maximum standardised uptake value

TRG tumour regression grade

QUADAS Quality Assessment of Studies of Diagnostic Accuracy Included in Systematic Reviews

$\mathrm{Cl} \quad$ Confidence interval

ROC Receiver operator curve

SROC Summary ROC

\section{Declarations}

Ethics approval and consent to participate 
Not applicable

\section{Consent for publication}

Not applicable

\section{Availability of data and materials}

The datasets during and/or analysed during the current study will be made available from the corresponding author on reasonable request.

\section{Competing interests}

The authors declare they have no competing interests.

\section{Funding}

The study has not received direct funding for its completion. KF receives funding from the Moondance Foundation at Velindre Cancer Centre.

\section{Sponsor}

Velindre University NHS Trust. The funder nor the sponsor have not been involved in the study design or protocol development and will not contribute to data collection, analysis or publication.

\section{Authors' contributions}

$\mathrm{JJ}$ participated in pilot data extraction and helped draft the manuscript. BC designed the search strategy. KB, ESmyth and ESpezi contributed to study design and manuscript preparation. AH provided statistical advice and contributed to data synthesis design. KF conceived the study idea, drafted the manuscript and is guarantor of the review. All authors read and approved the final manuscript.

\section{Acknowledgements}

The authors would like to thank the Oesophageal Cancer Clinical and Molecular Stratification (OCCAMS) consortium for their contribution to the systematic review concept and design.

\section{References}

1. Arnold M, Soerjomataram I, Ferlay J, Forman D. Global incidence of oesophageal cancer by histological subtype in 2012. Gut. 2015;64:381-7.

2. Cunningham D, Allum WH, Stenning SP, Thompson JN, Van de Velde CJ, Nicolson M, et al. Perioperative chemotherapy versus surgery alone for resectable gastroesophageal cancer. $\mathrm{N}$ Engl $\mathrm{J}$ Med. 2006;355:11-20. 
3. Crosby TD, Evans M. The management of locally advanced carcinoma of the oesophagus - SCOPE for improvement? Clin Oncol. 2009;21:281-4.

4. Sjoquist KM, Burmeister BH, Smithers BM, Zalcberg JR, Simes RJ, Barbour A, et al. Survival after neoadjuvant chemotherapy or chemoradiotherapy for resectable oesophageal carcinoma: an updated meta-analysis. Lancet Oncol. 2011;12:681-92.

5. Medical Research Council Oesophageal Cancer Working Group. Surgical resection with or without preoperative chemotherapy in oesophageal cancer: a randomised controlled trial. Lancet. 2002;359:1727-33.

6. Allum WH, Stenning SP, Bancewicz J, Clark PI, Langley RE. Long-term results of a randomized trial of surgery with or without preoperative chemotherapy in esophageal cancer. J Clin Oncol. 2009;27:5062-7.

7. Ychou M, Boige V, Pignon JP, Conroy T, Bouche O, Lebreton G, et al. Perioperative chemotherapy compared with surgery alone for resectable gastroesophageal adenocarcinoma: an FNCLCC and FFCD multicenter phase III trial. J Clin Oncol. 2011;29:1715-21.

8. van Hagen P, Hulshof MCCM, van Lanschot JJB, Steyerberg EW, van Berge Henegouwen MI, Wijnhoven BPL, et al. Preoperative chemoradiotherapy for esophageal or junctional cancer. N Engl J Med. 2012;366:2074-84.

9. Shapiro J, van Lanschot JJ, Hulshof MC, van Hagen P, van Berge Henegouwen MI, Wijnhoven BP, et al. Neoadjuvant chemoradiotherapy plus surgery versus surgery alone for oesophageal or junctional cancer (CROSS): long-term results of a randomised controlled trial. Lancet Oncol. 2015;16:1090-8.

10. Al-Batran S-E, Homann N, Pauligk C, Goetze TO, Meiler J, Kasper S, et al. Perioperative chemotherapy with fluorouracil plus leucovorin, oxaliplatin, and docetaxel versus fluorouracil or capecitabine plus cisplatin and epirubicin for locally advanced, resectable gastric or gastro-oesophageal junction adenocarcinoma (FLOT4). Lancet. 2019;393:1948-57.

11. Allum WH, Blazeby JM, Griffin SM, Cunningham D, Jankowski JA, Wong R. Guidelines for the management of oesophageal and gastric cancer. Gut. 2011;60:1449-72.

12. Gillies RS, Middleton MR, Maynard ND, Bradley KM, Gleeson F V. Additional benefit of (1)(8)Ffluorodeoxyglucose integrated positron emission tomography/computed tomography in the staging of oesophageal cancer. Eur Radiol. 2011;21:274-80.

13. Cremonesi M, Garibaldi C, Timmerman R, Ferrari M, Ronchi S, Grana CM, et al. Interim (18)F-FDG$\mathrm{PET} / \mathrm{CT}$ during chemo-radiotherapy in the management of oesophageal cancer patients: a systematic review. Radiother Oncol. 2017;125:200-12.

14. Zhu Y, Sun Y, Hu S, Jiang Y, Yue J, Xue X, et al. Comparison of five tumor regression grading systems for gastric adenocarcinoma after neoadjuvant chemotherapy: a retrospective study of 192 cases from National Cancer Center in China. BMC Gastroenterol. 2017;17:41.

15. Becker K, Mueller JD, Schulmacher C, Ott K, Fink U, Busch R, et al. Histomorphology and grading of regression in gastric carcinoma treated with neoadjuvant chemotherapy. Cancer. 2003;98:1521-30. 
16. Mandard AM, Dalibard F, Mandard JC, Marnay J, Henry-Amar M, Petiot JF, et al. Pathologic assessment of tumor regression after preoperative chemoradiotherapy of esophageal carcinoma. Clinicopathologic correlations. Cancer. 1994;73:2680-6.

17. Noble F, Lloyd MA, Turkington R, Griffiths E, O'Donovan M, O'Neill JR, et al. Multicentre cohort study to define and validate pathological assessment of response to neoadjuvant therapy in oesophagogastric adenocarcinoma. Br J Surg. 2017;104:1816-28.

18. Wahl RL, Jacene H, Kasamon Y, Lodge MA. From RECIST to PERCIST: Evolving Considerations for PET Response Criteria in Solid Tumours. J Nucl Med. 2009;50 Suppl 1:122S-50S.

19. Weber WA, Ott K, Becker K, Dittler HJ, Helmberger H, Avril NE, et al. Prediction of response to preoperative chemotherapy in adenocarcinomas of the esophagogastric junction by metabolic imaging. J Clin Oncol. 2001;19:3058-65.

20. Ott K, Weber WA, Lordick F, Becker K, Busch R, Herrmann K, et al. Metabolic imaging predicts response, survival, and recurrence in adenocarcinomas of the esophagogastric junction. $\mathrm{J}$ Clin Oncol. 2006;24:4692-8.

21. Lordick F, Ott K, Krause BJ, Weber WA, Becker K, Stein HJ, et al. PET to assess early metabolic response and to guide treatment of adenocarcinoma of the oesophagogastric junction: the MUNICON phase II trial. Lancet Oncol. 2007;8:797-805.

22. Shamseer L, Moher D, Clarke M, Ghersi D, Liberati A, Petticrew M, et al. Preferred reporting items for systematic review and meta-analysis protocols (PRISMA-P) 2015: elaboration and explanation. BMJ. 2015;350:g7647.

23. Wells G, Shea B, O'Connell D, Peterson J, Welch V, Losos M, et al. The Newcastle-Ottawa Scale (NOS) for assessing the quality of nonrandomised studies in meta-analyses. 2016. http://www.ohri.ca/programs/clinical_epidemiology/oxford.asp. Accessed 5 Aug 2020.

24. Whiting PF, Rutjes AWS, Westwood ME, Mallett S, Deeks JJ, Reitsma JB, et al. QUADAS-2: a revised tool for the quality assessment of diagnostic accuracy studies. Ann Intern Med. 2011;155:529-36.

25. Guyatt GH, Oxman AD, Vist GE, Kunz R, Falck-Ytter Y, Alonso-Coello P, et al. GRADE: an emerging consensus on rating quality of evidence and strength of recommendations. BMJ. 2008;336:924926.

26. DerSimonian R, Laird N. Meta-analysis in clinical trials. Control Clin Trials. 1986;7:177-88.

27. R Core Team. R: A language and environment for statistical computing. 2018. https://www.rproject.org/.

28. Moses LE, Shapiro D, Littenberg B. Combining independent studies of a diagnostic test into a summary ROC curve: data-analytic approaches and some additional considerations. Stat Med. 1993;12:1293-316.

29. Higgins JPT, Thompson SG, Deeks JJ, Altman DG. Measuring inconsistency in meta-analyses. BMJ. 2003;327:557-60.

30. Easterbrook PJ, Berlin JA, Gopalan R, Matthews DR. Publication bias in clinical research. Lancet. 1991;337:867-72. 
31. Kinahan PE, Fletcher JW. Positron emission tomography-computed tomography standardized uptake values in clinical practice and assessing response to therapy. Semin Ultrasound CT MR. 2010;31:496-505.

32. Kwee RM. Prediction of tumor response to neoadjuvant therapy in patients with esophageal cancer with use of 18F FDG PET: a systematic review. Radiology. 2010;254:707-17.

\section{Figures}




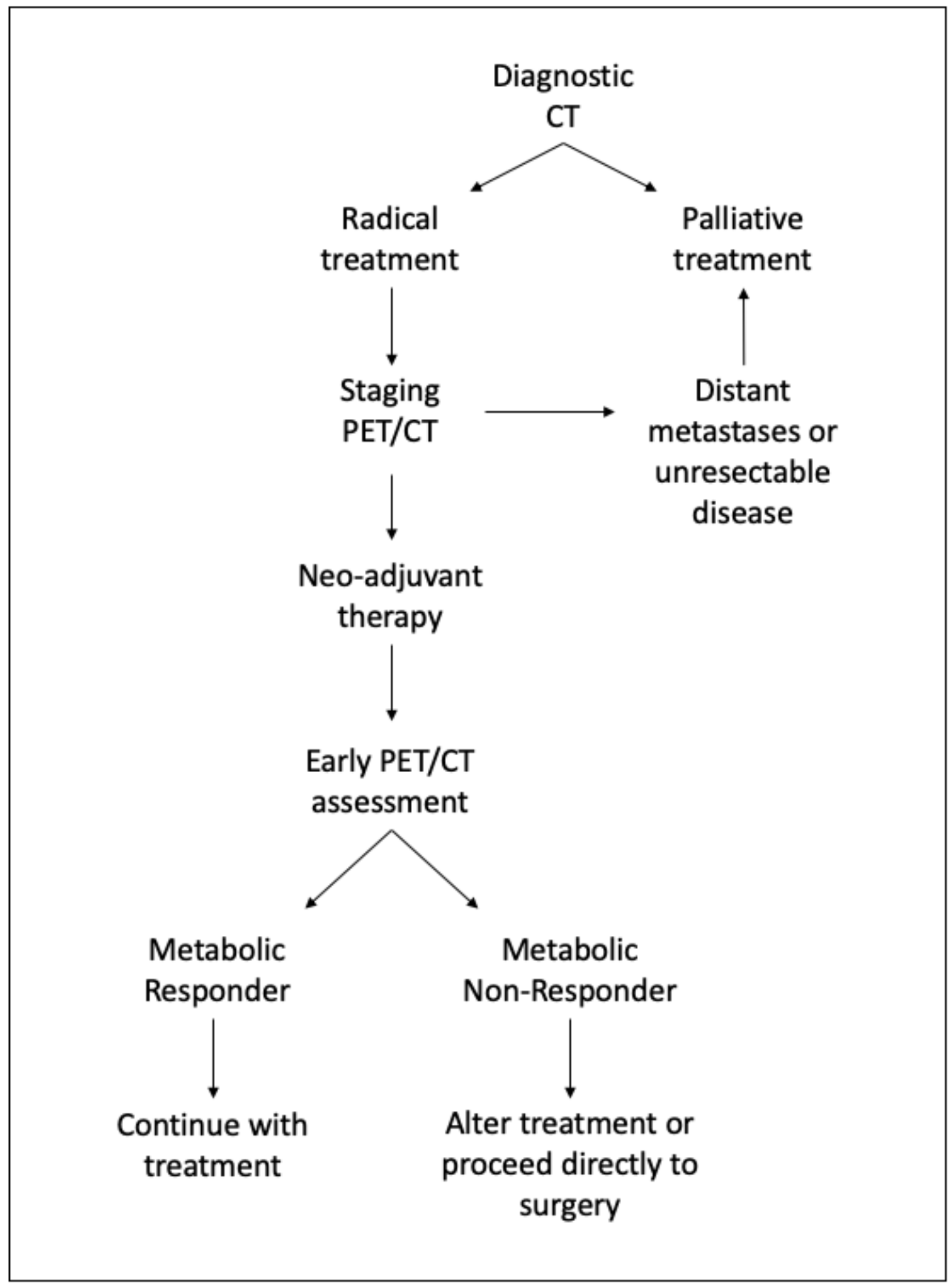

Figure 1

A schematic representation of the oesophageal cancer staging and treatment pathway, including early $\mathrm{PET} / \mathrm{CT}$, and the effect early PET/CT could have on clinical management.

\section{Supplementary Files}


This is a list of supplementary files associated with this preprint. Click to download.

- Additionalfile1PRISMAPChecklist.docx

- Additionalfile2SystRevDataltemsExtraction.xlsx

- Additionalfile3ModifiedNOS.xlsx

- Additionalfile4QUADAS2.docx 